\title{
RELACIÓN ENTRE MALOCLUSIONES Y AUTOPERCEPCIÓN DE LA NECESIDAD DEL TRATAMIENTO ORTODÓNTICO SAN SEBASTIÁN CUENCA 2016
}

\section{RELATIONSHIP BETWEEN MALOCLUSIONS AND SELF-IMPROVEMENT OF THE NEED FOR ORTHODONTIC TREATMENT SAN SEBASTIÁN CUENCA 2016}

\author{
Jiménez-Patiño Jhoana $^{1 *}$, Ramos-Montiel Ronald ${ }^{2}$ \\ 1 Odontóloga de la Universidad Católica de Cuenca. Ecuador \\ ${ }^{2}$ Catedrático de la Carrera de Odontología de la Universidad Católica de Cuenca. Ecuador \\ *j_ho_ana@hotmail.es
}

\begin{abstract}
Resumen
OBJETIVO: Relacionar la prevalencia de maloclusiones y la necesidad de tratamiento ortodóntico en escolares de 12 años en la parroquia San Sebastián cantón Cuenca año 2016. MATERIALES Y MÉTODOS:: Se realizó un estudio cuantitativo, el diseño fue descriptivo y la técnica observacional, retrospectiva. La muestra estuvo constituida por 245 escolares de 12 años, la metodología que se aplicó fue cuantitativa, el diseño fue descriptivo y la técnica observacional, retrospectiva. La muestra estuvo constituida por 245 escolares de 12 años. RESULTADOS: Existió prevalencia de maloclusiones con el $97 \%$ con respecto a la normooclusión representada por el 3\%; según Angle en el sexo femenino existió mayor frecuencia de Clase II, luego de Clase I y con menos frecuencia de Clase III. En el sexo masculino existió mayor frecuencia de Clase I, seguida de Clase II y con menos frecuencia la Clase III. Según la autopercepción y necesidad de tratamiento ortodóntico el sexo masculino es predominante el 4,5\% autopercibieron que tienen necesidad evidente, el 71,8\% autopercibieron que no necesitan y el 20,4\% autopercibieron necesidad moderada; en el sexo femenino prevalecieron las maloclusiones. En ambos sexos existió un porcentaje mayor de los escolares que autopercibieron que no necesitan, seguido de los que autopercibieron necesidad moderada y por último los que autopercibieron que tienen necesidad evidente de tratamiento ortodóntico. CONCLUSIÓN: De acuerdo a la relación de la prevalencia de maloclusiones y la autopercepción de la necesidad de tratamiento ortodóntico de los escolares de 12 años se observó que el 4,5\% autopercibieron que tienen necesidad evidente, el 71,8\% autopercibieron que no necesitan y el 20,4\% autopercibieron que tienen necesidad moderada de tratamiento ortodóntico.
\end{abstract}

Palabras clave: Maloclusión, Normoclusión, Prevalencia, Epidemiología.

\begin{abstract}
OBJECTIVE:To relate the prevalence between malocclusions and self-perception of the need for orthodontic treatment of 12year-old schoolchildren from the San Sebastian parish of Cuenca, year 2016. MATERIALS AND METHODS:A quantitative study was carried out, the design was descriptive and the observational technique was retrospective. The sample consisted of 245 schoolchildren aged 12 years, the methodology applied was quantitative, the design was descriptive and the observational technique was retrospective. The sample consisted of 245 schoolchildren aged 12 years. RESULTS: There was a prevalence of malocclusions with 97\% with respect to the normooclusion represented by 3\%; According to Angle in the female sex there was a higher frequency of Class II, followed by Class I and less frequently Class III. In the male sex, there was a higher frequency of Class I, followed by Class II and less frequently Class III. According to the self-perception and need for orthodontic treatment, the male gender is predominant, $4.5 \%$ self-perceived that they have evident need, $71.8 \%$ self-perceived that they do not need and 20.4\% self-perceived moderate need; Malocclusions prevailed in the female sex. In both sexes there was a higher percentage of students who self-perceived that they did not need, followed by those who self-perceived moderate need and finally those who self-perceived that they had evident need for orthodontic treatment. CONCLUSIONS: According to the relationship between the prevalence of malocclusions and the self-perception of the need for orthodontic treatment of 12-year-old schoolchildren, it was observed that 4.5\% self-perceived that they have evident need, $71.8 \%$ self-perceived that they do not need and 20.4\% self-perceived that they have moderate need for orthodontic treatment.
\end{abstract}

Key words: Malocclusion, Normocclusion, Prevalence, Epidemiology. 


\section{INTRODUCCIÓN}

Las maloclusiones dentales son un problema dentro de la sociedad que ha aumentado de manera progresiva, al hablar de maloclusión se debe entender que es la mal posición o posición incorrecta de los dientes dentro de los arcos dentales como es el maxilar superior y el maxilar inferior, al existir mal posición dental se va a encontrar deficiencias en las funciones fonéticas, deglutorias y sobre todo en la estética facial que se va a ver alterada. "La Organización Mundial de la Salud (OMS), indica valorar esta enfermedad en pacientes de 6, 12 y 18 años", pero la siguiente investigación se basará en escolares de 12 años. ${ }^{1-3}$

Muchas de las maloclusiones se pueden prevenir o tratar en sus etapas iniciales, para prevenirla se requiere total colaboración del paciente y que el profesional de la salud dental realice un correcto diagnóstico y por ende un buen tratamiento. La autoestima y autopercepción del paciente también juegan un papel importante para lograr lo antes mencionado y saber si está apto o no para recibir un tratamiento.

Dentro de la autoestima de una persona la principal consideración es la belleza facial, al existir una proporción divina en el rostro le otorga belleza y armonía, pero dentro de ortodoncia es el índice facial el que dicta normas para que las dimensiones de la cara sean armónicas. Sin embargo, no está despejada la relación entre estas mediciones objetivas y la autopercepción estética que tienen las personas de su propio atractivo facial. ${ }^{4,5}$ Las personas con autoestima baja subestiman mucho su aspecto dental al ser comparadas con personas que tienen la autoestima alta sin darle valor al tipo de oclusión que presentan. ${ }^{6-8}$

La autopercepción de las personas son indicadores significativos para saber sobre la necesidad de tratamiento y mejoramiento de las medidas convencionales. Los indicadores sociales permiten dar prioridad a las personas que necesitan el tratamiento separando de la limitación de los recursos financieros para mejorar el bienestar social y psicológico. ${ }^{9,10}$

La presente investigación brindó información sobre la relación de la prevalencia de las maloclusiones dentales y necesidad del tratamiento ortodóntico autopercibido en escolares de 12 años de edad de la parroquia San Sebastián perteneciente al cantón Cuenca, la misma que se basó en dos componentes que son, el componente de salud dental (CSD) y el componente estético (CE), para establecer el CSD se manejó la observación clínica intraoral y para el CE el escolar evaluado enseñó por sí mismo en que nivel de puntuación del 1 al 10 se encuentra según la fotografía que se le hizo observar. ${ }^{11,12}$

\section{MATERIALES Y METODOS}

Este estudio es descriptivo, transversal retrospectivo de análisis cuantitativo. La totalidad de la muestra corresponde a 245 escolares de 12 años de edad de la parroquia San Sebastián de la ciudad de Cuenca-Ecuador. Empleándose los siguientes criterios de inclusión: estudiantes de ambos sexos que se encuentren matriculados en las unidades pedagógicas de la parroquia, que posean 12 años cumplidos o que vayan a cumplir hasta el 31 de diciembre del 2016 y que estén en la oficina de investigación de la Universidad Católica de Cuenca, la base de datos se obtuvo por medio del programa Epi-Info, en el cual se seleccionó las variables que se plantearon analizar. Los datos fueron analizados mediante el programa Excel 2013 para conseguir gráficos estadísticos.

Para evaluar el componente estético se utilizó fotos intraorales de 100 niños de 12 años en un estudio multidisciplinario. En donde 6 jurados que no tenían ninguna relación con odontología puntuaron las fotografías en una escala visual análoga y escogieron algunas fotos que representaban 10 intervalos, obteniendo escalas de 10 puntos, donde el número 1 significa una atractiva estética dental y la 10 significa que no es atractiva. ${ }^{13,14}$

Para el inicio del estudio se solicitó el consentimiento de la institución educativa y que presenten el consentimiento informado de sus padres y/o asentimiento del escolar.Todos los sujetos fueron examinados visual y clínicamente por un grupo de 8 examinadores capacitados y estandarizados en el criterio diagnóstico de maloclusiones, utilizando para la medición de las variables clínicas un espejo bucal, luz natural y una paleta baja lenguas. Los examinadores emplearon guantes, mascarilla y vestimenta clínica, además de esterilizar los instrumentos previamente.

\section{RESULTADOS}

Este estudio se realizó con los datos Epidemiológicos de los escolares de la Parroquia San Sebastián, que están en el archivo del departamento de investigación de la carrera de Odontología de la UCACUE.

El sexo masculino es predominante en los escolares de 12 años de la parroquia San Sebastián. (Gráfico N 1)

La presencia de maloclusiones y la autopercepción de la necesidad de tratamiento ortodóntico, observándose que dentro de los escolares que presentan maloclusiones, el 4,5\% autopercibieron que tienen necesidad evidente de tratamiento, el $71,8 \%$ autopercibieron que no necesitan tratamiento y el $20,4 \%$ autopercibieron que tienen necesidad moderada de tratamiento ortodóntico. En relación a los pacientes que presentaron oclusión normal el 2,9\% autopercibieron que no necesitan tratamiento, mientras que el $0,4 \%$ autopercibieron que tienen una necesidad moderada de tratamiento ortodóntico. (Gráfico N 2) 
Grf. 1. Distribución de escolares de 12 años de la parroquia San Sebastián según el sexo.

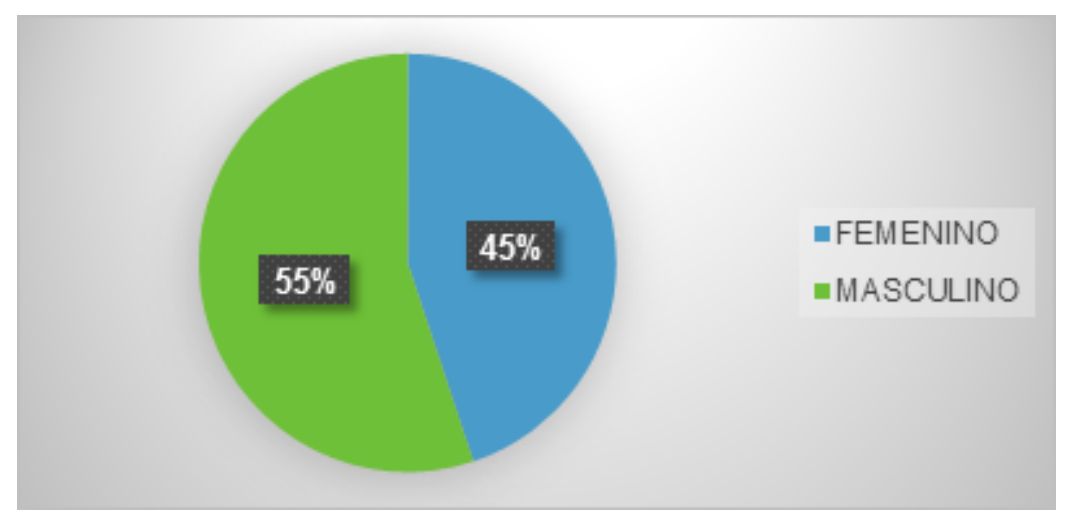

Grf. 2. Relación entre la presencia de maloclusiones y la autopercepción de la necesidad de tratamiento ortodóntico (IONT) de acuerdo al sexo en escolares de 12 años de la parroquia San Sebastián.

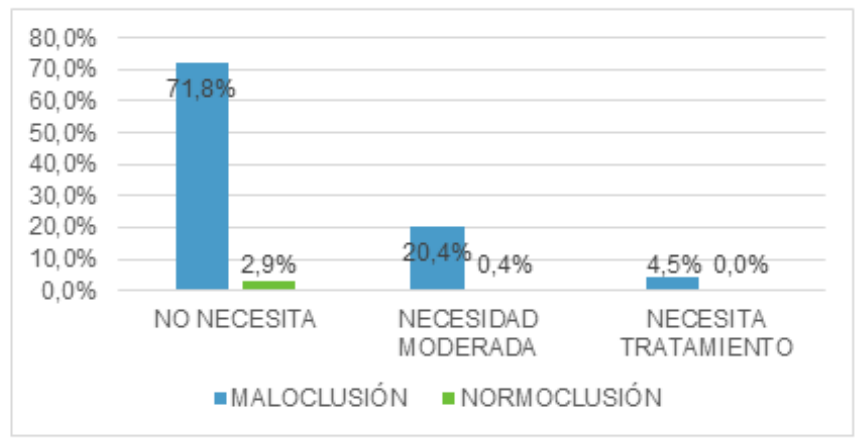

Existe mayor prevalencia de maloclusiones con el $97 \%$, con respecto a la normoclusión representada por el $3 \%$ de la muestra estudiada. (Gráfico N 3)

La prevalencia de maloclusiones es mayor en el sexo femenino que en el sexo masculino. (Gráfico N 4)

Tanto en el sexo masculino como femenino existe un porcentaje mayor de los escolares que autopercibieron que no necesitan tratamiento, seguido de la necesidad moderada de tratamiento y por último los que tienen necesidad evidente de tratamiento ortodóntico. (Gráfico N 5)

En el sexo femenino existe mayor frecuencia de la Clase II con el $23 \%$, seguida por la Clase I con el $12 \%$ y con menor frecuencia la Clase III con el $9 \%$, mientras que en el sexo masculino existe una frecuencia similar de la Clase I y Clase II con el $20 \%$ cada una y finalmente con menor frecuencia la Clase III con el $13 \%$. (Gráfico N 6)

\section{DISCUSIÓN}

Mediante el estudio realizado en la Amazonia de Ucayali, Perú se descubrió que la mayor parte de la población atendida tenían maloclusiones en un porcentaje de $85,6 \%$. $^{5}$ Teniendo gran similitud con el estudio epidemiológico realizado en el año 2016 en Ecuador en la parroquia de San
Sebastián del cantón Cuenca en donde igual existió mayor prevalencia de maloclusiones con el $97 \%$, con respecto a la normoclusión representada por el $3 \%$.

Según Almerich J, Montiel J. en el estudio elaborado en la comunidad Valenciana tomando como referencia el IONT, el $20,9 \%$ de los niños de 12 años requieren tratamiento ortodóntico $^{15,16} \mathrm{Al}$ revisar este estudio Cuenca muestra que se separó la necesidad de tratamiento de acuerdo al sexo mostrando así que del sexo femenino el $2 \%$ perciben que necesitan tratamiento, mientras que del sexo masculino el $7 \%$ perciben que lo necesitan.

Según el artículo de Rodríguez M, Parrón T, Nieto J donde se realizó un estudio observacional descriptivo o de prevalencia mediante un muestreo aleatorio simple en colegios de 220 escolares de 12 y 15 años. El $67.1 \%$ de la población tenía un grado de Índice estético dental mayor o igual a 25. Resaltamos varias alteraciones, donde el $10 \%$ de la población tiene un grado de Índice estético dental menor o igual a 36 catalogándola como poseedora de una maloclusión severa que necesita tratamiento y poder utilizar estos parámetros como indicadores para dar preferencia a la población que necesita tratamiento. ${ }^{17,18}$ Comparando con 
Grf. 3. Prevalencia de las maloclusiones de los escolares de 12 años de edad de la parroquia San Sebastián.

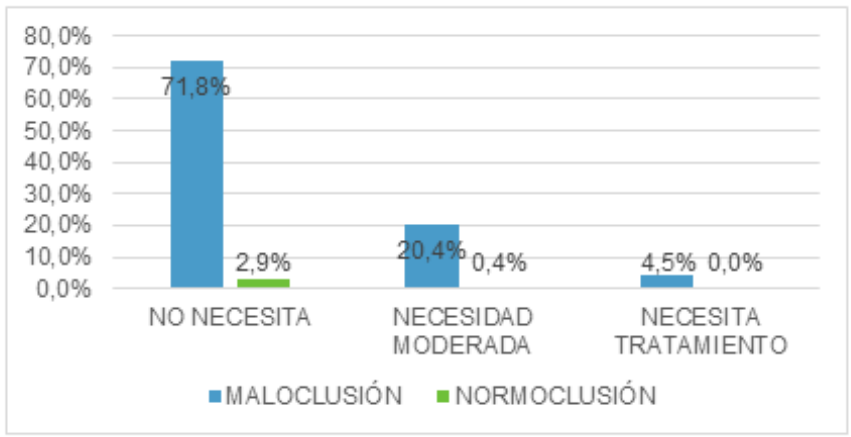

Grf. 4. Prevalencia de maloclusiones según el sexo en los escolares de 12 años de la parroquia San Sebastián.

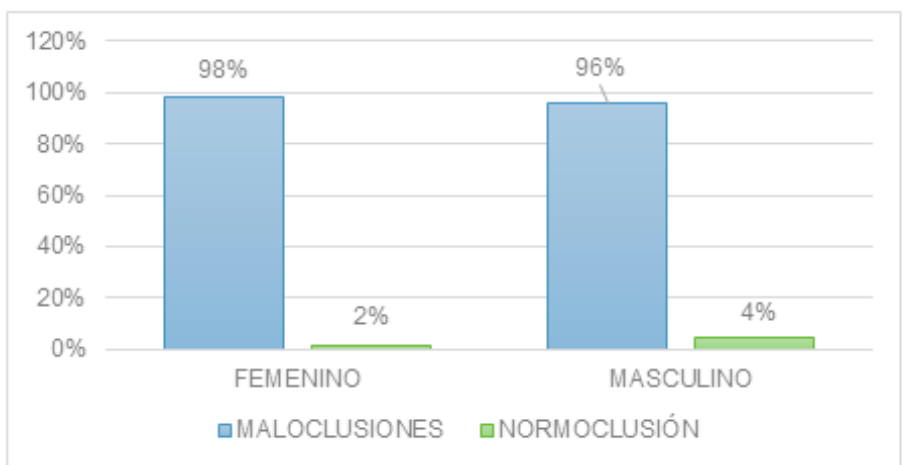

Grf. 5. Necesidad de tratamiento ortodóntico autopercibido (IONT) de acuerdo al sexo en escolares de 12 años de la parroquia San Sebastián.

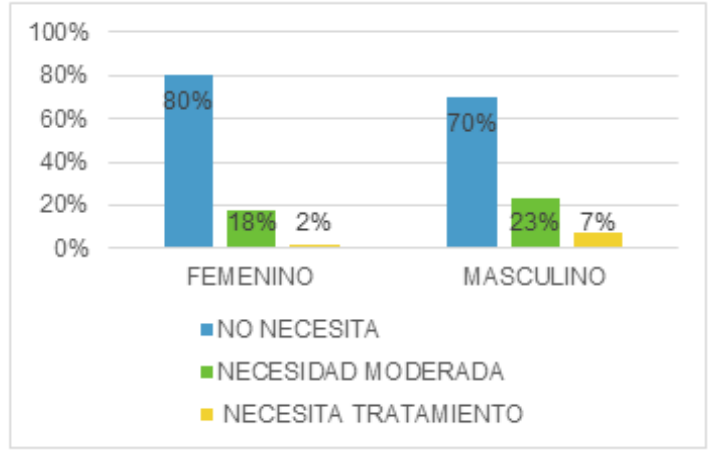

el actual estudio en donde para saber la autopercepción y necesidad de tratamiento ortodóntico se utilizó una fotografía con intervalos del 1 al 10 distribuidos en 3 escalas: del 1 a 4 no necesitan tratamiento, del 5 a 7 necesidad moderada y de 6 a 10 necesidad evidente de tratamiento, con todo esto se obtuvo los siguientes resultados el $97 \%$ presentaron maloclusiones el 4,5\% necesitan tratamiento, el $71,8 \%$ no necesitan y el $20,4 \%$ tienen necesidad moderada. Mientras que en la normoclusión el 2,9\% no necesita y el 0,4\% tienen necesidad moderada de tratamiento.
La clasificación de Angle es bastante utilizada por la simplicidad que presenta dentro de la cual tenemos tres grupos con subdivisiones. ${ }^{19}$ Con respecto a esto tenemos un estudio realizado en la Amazonia de Ucayali, Perú en una población de 372 niños en donde el 85,6 presento maloclusiones siendo la clase I más habitual en ambos sexos con $59,6 \%$, a continuación va la clase II con $18,5 \%$, la clase III se encontró con menor frecuencia en ambos sexos 9,2. ${ }^{20}$ A comparación con las deducciones emanados en el estudio epidemiológico realizado en el año 2016 en Ecuador 
Grf. 6. Frecuencia de maloclusiones según Angle de acuerdo al sexo en escolares de 12 años de la parroquia San Sebastián.

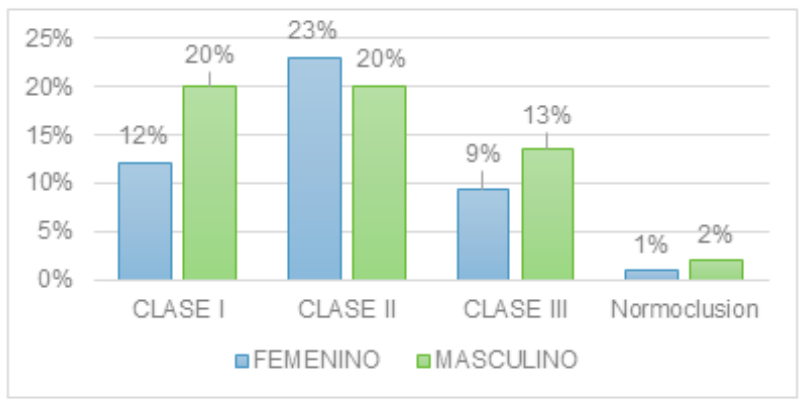

en la parroquia de San Sebastián del cantón Cuenca donde arrojó que la frecuencia varía de acuerdo al sexo, en el sexo femenino existe mayor frecuencia de la clase II, seguida por la clase I y con menor frecuencia la clase III. Mientras que en el sexo masculino existe una frecuencia similar de la clase I y clase II y finalmente con menor frecuencia la clase III. En un estudio realizado en México por Murrieta $\mathrm{J}$ y col, evidenció que Clase I de Angle la más frecuente (72,8\%), La Clase II fue la segunda más frecuente en el 13,5\% de los casos detectados con maloclusión y finalmente la Clase III en el 10,1\%.$^{21}$ En el estudio epidemiológico realizado en la parroquia de San Sebastián donde arrojo que hay diferentes frecuencias de acuerdo al sexo siendo así que el sexo femenino existe mayor frecuencia de la clase II (23\%), seguida por la clase I $(12 \%)$ y con menor frecuencia la clase III (9\%). Mientras que en el sexo masculino existe una frecuencia similar de la clase I y clase II $(20 \%)$ y finalmente con menor frecuencia la clase III $(13 \%)$.

\section{CONCLUSIÓN}

La relación entre la presencia de maloclusiones y la autopercepción de la necesidad de tratamiento ortodóntico de los escolares de 12 años se observó que el 4,5\% autopercibieron que tienen necesidad evidente, el 71,8\% autopercibieron que no necesitan y el $20,4 \%$ autopercibieron que tienen necesidad moderada de tratamiento ortodóntico. La prevalencia de las maloclusiones es mayor representada por el $97 \%$ con respecto a la normoclusión que está representada por el $3 \%$ de la población. La prevalencia de las maloclusiones de acuerdo al sexo en los escolares fueron bastante elevadas, pero el sexo más afectado fue el femenino. En la autopercepción de la necesidad de tratamiento ortodóntico tanto el sexo masculino como femenino obtuvieron un porcentaje mayor de los escolares que autopercibieron que no necesitan, seguido de la necesidad moderada y por último que tienen necesidad evidente de tratamiento ortodóntico. Las maloclusiones según Angle la que se presenta con mayor frecuencia varía de acuerdo al sexo, en el sexo femenino existe mayor frecuencia de la Clase II, seguida por la Clase I y con menor frecuencia la Clase III, mientras que en el sexo masculino existe mayor frecuencia de la Clase I y la Clase II y con menor frecuencia la Clase III. Promover la salud bucal de los pacientes y de la población en general. Consiste en educar desde la perspectiva sanitaria e higiénica. En él también se incluye el conocimiento los factores generales que influyen en el desarrollo de las maloclusiones: factores hereditarios, en el embarazo, en el parto y después del nacimiento.

Conflicto de intereses y financiamiento Los autores declaran no tener conflicto de intereses, haber cumplido con los requisitos de autoría y haber autofinanciado este artículo.

\section{Referencias}

1 Di Santi de Modano J, Vázquez V. Maloclusión Clase I: Definición, clasificación, características clínicas y tratamiento. Rev Lat de Ort y Odontopeditr [Internet]. 2003 [citado 22 Nov 2016]; 1-6. Documento disponible

2 Almerich J, Montiel J. Puertes N. Necesidad de tratamiento ortodóncico e impacto psicosocial de la estética dental en los adolescentes de la comunidad valenciana (2010) [Internet]. 2013- Noviembre [citado 23 Nov 2016]; 1836.Documento disponible

3 Bustamante G, Surco V, Tito E, Yujra C. Oclusión. Rev Boliv [Internet]. 2012 Mayo [citado 22 Nov 2016]; 20. Documento disponible

4 Bonilla A, Rosell L. Clasificación de las maloclusiones. Scribd [Internet]. [citado 24 Nov 2016]; 3-6, 32-59. Documento disponible

5 Aligaga A, Mattos M, Aliaga R, Mendoza C. Maloclusiones en niños y adolescentes de caseríos y comunidades nativas de la Amazonía de Ucayali, Perú. Rev. Perú. med. exp. salud publica SciELO. [Internet]. 2011 Marzo [citado 21 Nov 2016]; 28(1). Documento disponible

6 Cuoghi O, De Mendonça M, Miranda Y. Mordida cruzada posterior. Corrección y consideraciones. Caso clínico con 7 años de seguimiento. Acta Odontológica Venezolana. 
[Internet] 2011 [citado 23 Nov 2016]; 49(1). Documento disponible

7 Castañer Amparo. Ortodoncia interceptiva: Necesidad de diagnóstico y tratamiento temprano en las mordidas cruzadas transversales. Medicina oral [Internet]. 2006 [citado 22 Nov 2016]; 211-213 Documento disponible

8 Jiménez V, González E, Contasti G. Influencia de la Mordida Cruzada posterior Unilateral en el crecimiento mandibular. Rev Latinoamericana de ortodoncia y odontopediatría [Internet]. 2006 [citado 24 Nov 2016]. Documento disponible

9 Cruz Irene. Corrección no quirúrgica de una mordida en tijera unilateral en un paciente CII división 2 con mordida profunda [Internet]; 2008 [citado 22 Nov 2016];1-5. Documento disponible

10 Sada M, De Girón J. Maloclusiones en la dentición temporal o mixta. An Pediatr Cont. [Internet]; 2006 [citado 23 Nov 2016]; 4 (1). Documento disponible

11 Alarcón A, Andrea M. Etiología, diagnóstico y plan de tratamiento de la mordida profunda - Revisión de la literatura. Rev Lat de Ort y Odontopeditr [Internet]. 2014 [citado 21 Nov 2016]. Documento disponible

12 Alcaraz C, Bordón J, Ríos D. Frecuencia de Mordida Abierta Anterior en escolares del Primero al Noveno Grado y sus factores asociados Pediatr. (Asunción) SciELO [Internet]. 2012 [citado 23 Nov 2016]; 39(2). Documento disponible

13 Sosa J. Rodriguez M. "Maloclusión (clasificación según Angle) en alumnos de segundo y tercer semestre de la facultad de odontología de la Universidad Central del Ecuador. Quito - Ecuador. Marzo-julio. 2012" Repositorio Dig [Internet] 2012 [citado 24 Nov 2016]. Documento disponible

14 Gutiérrez D, Díaz R, Valenti M. Prevalencia de Mordida cruzada dental en pacientes pediátricos de la Clínica Periférica "Las Águilas"turno vespertino de octubre 2005 a marzo 2006. Rev Lat de Ort y Odontopediatr [Internet]. 2007 [citado 25 Nov 2016]. Documento disponible

15 Tosta O. Análisis facial en la estética dental. Gaceta Dental [Internet]. 2011 [citado 22 Nov 2016]. Documento disponible

16 Singh Gurkeerat. Ortodoncia Diagnóstico y Tratamiento. Tomo 1. Segunda Edición ed. Singh G, editor. USA: AMOLCA; 2009.

17 Hechavarria B, Venzant S, Carbonell MdlC, Carbonell C. Salud bucal en la adolescencia. MEDISAN SciELO [Internet].2013 [citado 24 Nov 2016]; 17(1). Documento disponible

18 Rodríguez M, Parrón T, Nieto J. Epidemiología de maloclusiones en niños de 12 y 15 años aplicando el índice estético dental. ScienceDirect [Internet].2003 [citado 25 Nov 2016]; 45: 94-101. Documento disponible

19 Murrieta J, Cruz P, López J, Marques Dos Santos M, Zurita V. Prevalencia de maloclusiones dentales en un grupo de adolescentes mexicanos y su relación con la edad y el género. Acta Odontológica Venezolana [Internet]. 2007 Enero [citado 28 Dic 2016]; 45(1). Documento disponible

20 Villavicencio E. El tamaño muestral en tesis de post grado. ¿cuantas personas debo encuestar? ResearchGate [Internet]. 2011 Enero [citado 30 Dic 2016]; 1-4. Documento disponible

21 Villavicencio E, Cuenca K, Vélez E, Sayago J, Cabrera A. Pasos para la planificación de una investigación clínica. ODONTOLOGIA activa UCACUE [Internet]. 2016 [citado 30 Dic 2016]; 1(1): 1-5. Documento disponible

Recibido: 20 de Enero de 2017

Aceptado: 30 de Marzo de 2017 